\title{
Risk assessment for public health from human interaction with ornamental waters
}

\author{
Aida Duarte ${ }^{1}$, João Carlos Rodrigues ${ }^{2}$, Lúcia Reis ${ }^{2}$, Isabel Nogueira ${ }^{3}$, Patricia Carvalho ${ }^{3}$, Sérgio \\ Paulino $^{4}$, Sara Sousa ${ }^{4}$ and Luisa Jordao ${ }^{4,5}$ \\ ${ }^{1}$ Departamento de Microbiologia e Imunologia, iMed.UL, Faculdade de Farmácia da Universidade de \\ Lisboa, Lisboa, Portugal. \\ ${ }^{2}$ Departamento de Doenças Infeciosas, Instituto Nacional de Saúde Dr Ricardo Jorge (INSA), Lisboa, \\ Portugal. \\ ${ }^{3}$ Departamento de Engenharia Química, Instituto Superior Técnico, Lisboa, Portugal. \\ ${ }^{4}$ Departamento de Saúde Ambiental (DSA), INSA, Lisboa, Portugal. \\ ${ }^{5}$ DSA, INSA, Avenida Padre Cruz 1649-016 Lisboa, Portugal.
}

Water is essential to life; nevertheless ingestion of contaminated water could result in death caused by waterborne diseases such as cholera [1]. Pathogens present in the water can cause diseases, other than those resulting from water ingestion, being registered an increase in the number of case reports in recent years. It is not clear if this increase is due either to a better case reporting system or to an increase in microorganism's virulence. The generalized use of antibiotics in agriculture and animal farming contributed to their dissemination in the environment which promotes microorganism selection and emergence of resistant strains. This phenomenon can be enhanced by the ability of microorganism to persist within complex communities known as biofilms.

In the present work we aim to characterize the microbial population present in ornamental waters and perform a risk assessment for public health resulting from human interaction with it.

Samples were collected in the Lisboa area between December 2014 and February 2015. Bacteria were identified either by growth on non-selective/ selective media incubated at different temperatures or VITEC after filtration through a $0.45 \mu \mathrm{m}$ membrane. Antibiotic susceptibility tests were performed and analyzed for planktonic bacteria according to EUCAST. Biofilm assembly, sample preparation for scanning electron microscopy (SEM) and minimal inhibitory concentration for amoxicillin (AMX) were performed as described previously [2].

In all water samples more than 300 colony forming units (CFU) per $10 \mathrm{~mL}$ were found on plate count agar. The majority of the identified microorganisms were members of Enterobacteriacea family namely, Klebsiella peneumoniae (Kp), K. pneumoniae ozaenae, Enterobacter spp., Serratia marcescens, S. rubidea e $S$. odorifera. Non fermentative, oxidase positive bacteria such as Elizabethkingia meningoseptica, Stenotrophomonas maltophilia and Vibrio metschnikovii were also identified.

The presence of a main etiological agent of healthcare associated infections (HAI) with high rates of antibiotic resistance among the isolates is an interesting and concerning result [3]. Since Kp resistance to antibiotics is linked to biofilm assembly [2] we decided to investigate the presence of Kp biofilms within the lakes. Kp biofilms were present only in lake 2. Further analysis of the Kp isolates rendered the results showed in table 1.

All the bacteria were able to assemble biofilms in conditions mimicking those present in the environment $\left(25^{\circ} \mathrm{C}\right)$. This ability is decreased at $37^{\circ} \mathrm{C}$ or even absent $(\mathrm{Kp} 2)$. Bacteria fitness to environmental conditions might suggest a diminish ability to colonize human beings. The environmental fitness was further 
documented by analysis of biofilms using SEM. The best organized biofilms were assembled on cement at $25^{\circ} \mathrm{C}$ (Fig. 1.A). Both temperature (Fig. 1.B) and surface (Fig. 1.C) changes lead to a decreased biofilm assembly.

In contrast to clinical isolates environmental $\mathrm{Kp}$ were susceptible to the 3 main groups of antibiotics: $\beta$ lactam, aminoglycosides and quinolones. In other words environmental $\mathrm{Kp}$ do not meet the criteria to be classified as multi-resistant bacteria. For this reason only biofilm susceptibility to amoxicillin was assayed. Biofilm assembly increased antibiotic resistance of Kp. Two isolates (Kp3 and 4) exhibited the same MIC value $(500 \mu \mathrm{g} / \mathrm{mL})$ which is significantly higher than the MICs founds for Kp2 $(0.98 \mu \mathrm{g} / \mathrm{mL})$ and Kp3 $(7.81 \mu \mathrm{g} / \mathrm{mL})$. This result together with the fact that Kp3 and 4 were isolated from the same lake lead us to hypothesize that the two isolates might be part of the same biofilm being the presence of $\mathrm{Kp} 3$ in the water explained by biofilm dispersion.

The low risk of environmental Kp for human health and the universal role played by biofilm assembly on antibiotic resistance are the main conclusions arising from this study.

\section{References:}

[1] J.P.S. Cabral, Int. J. Enviro Res Public Health, 7 (2010) p. 3657.

[2] M. Bandeira, et al., Pathogens, 3 (2014) p. 720.

[3] ECDC Europe, (2013), http://www.ecdc.europa.eu/en/publications/publications/annualepidemiological-report-2013.pdf
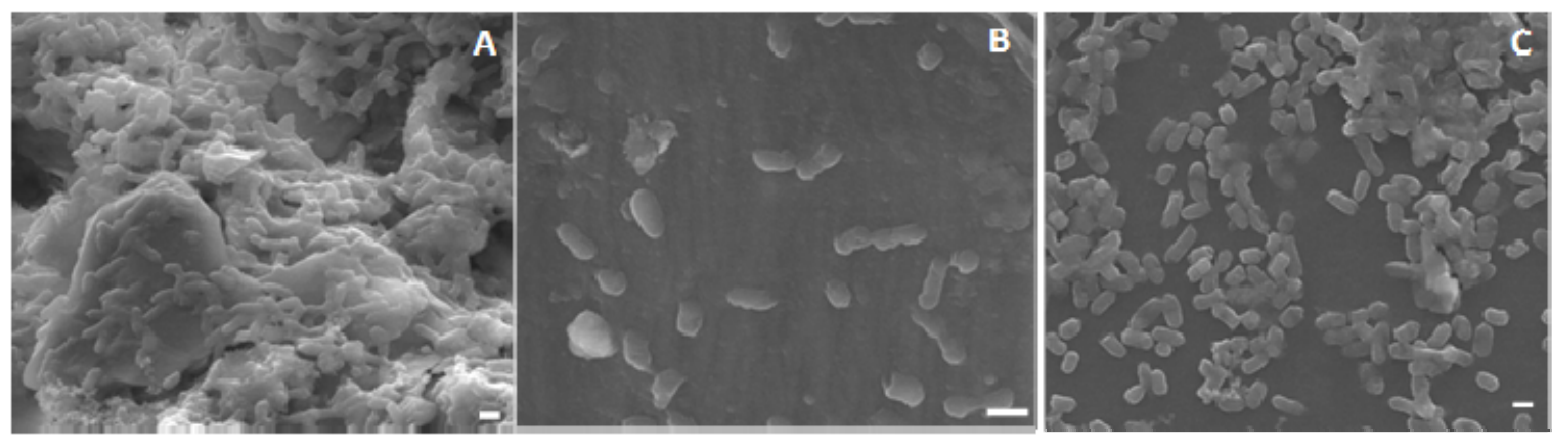

Figure 1. Klebsiella pneumonia biofilms. The ability of $\mathrm{Kp} 4$ to assemble biofilms in vitro on cement at $25^{\circ} \mathrm{C}(\mathrm{A})$ and $37^{\circ} \mathrm{C}(\mathrm{B})$ was evaluated. In order to evaluate the role played by surface on biofilm assembly glass was also used (C). All the biofilms were $48 \mathrm{~h}$ old. Scale bar $=1 \mu \mathrm{m}$.

Table 1. Characterization of Klebsiella pneumoniae isolates.

\begin{tabular}{|c|c|c|c|c|c|c|c|c|c|c|c|c|}
\hline \multirow[t]{2}{*}{ ID } & \multirow[t]{2}{*}{ Source } & \multicolumn{2}{|c|}{$\begin{array}{l}\text { Biofilm assay } \\
\text { (OD570nm) }\end{array}$} & \multicolumn{7}{|c|}{$\begin{array}{c}\text { Antibiogram } \\
(\mathrm{mm})\end{array}$} & \multicolumn{2}{|c|}{$\begin{array}{c}\text { MIC AMX } \\
(\mu \mathrm{g} / \mathrm{ml})\end{array}$} \\
\hline & & $25 \circ \mathrm{C}$ & $37^{\circ} \mathrm{C}$ & AMC & FOX & CAZ & CTX & IPM & GM & CIP & Plant & Biof \\
\hline Kp1 & Lake 1 & 0.240 & 0.153 & 18 & 28 & $>30$ & $>30$ & $>30$ & 24 & $>30$ & 7,81 & 62,5 \\
\hline Kp2 & Lake 2 & 0.076 & --- & 6 & 20 & 25 & 30 & 25 & 18 & 28 & 0,98 & 125 \\
\hline Kp3 & Lake2 & 0.740 & 0.256 & 6 & 24 & $>30$ & $>30$ & 22 & 18 & $>30$ & 500 & $>500$ \\
\hline Kp4 & Biof Lake 2 & 1.159 & 0.285 & 6 & 6 & 25 & 29 & 25 & 18 & 28 & 500 & $>500$ \\
\hline
\end{tabular}

Plant: Planktonic bactéria, Biof: biofilm; AMC: combination of amoxycillin and clavulanic acid; AMX: amoxycillin; FOX: cefoxitin; CAZ: ceftazidime; CTX: cefotaxime; IPM: imipenem; GM: gentamicin and CIP: ciprofloxacin. 